\title{
Role of the Inflammasome, IL-1 $\beta$, and IL-18 in Bacterial Infections
}

\author{
Manoranjan Sahoo, Ivonne Ceballos-Olvera, Laura del Barrio, and Fabio Re \\ Department of Microbiology, Immunology, and Biochemistry, University of Tennessee Health Science Center, \\ Memphis, TN 38163, USA
}

Received 20 July 2011; Accepted 30 September 2011

Academic Editor: Giamila Fantuzzi

The inflammasome is an important innate immune pathway that regulates at least two host responses protective against infections: (1) secretion of the proinflammatory cytokines IL- $1 \beta$ and IL-18 and (2) induction of pyroptosis, a form of cell death. Inflammasomes, of which different types have been identified, are multiprotein complexes containing pattern recognition receptors belonging to the Nod-like receptor family or the PYHIN family and the protease caspase-1. The molecular aspects involved in the activation of different inflammasomes by various pathogens are being rapidly elucidated, and their role during infections is being characterized. Production of IL-1 $\beta$ and IL-18 and induction of pyroptosis of the infected cell have been shown to be protective against many infectious agents. Here, we review the recent literature concerning inflammasome activation in the context of bacterial infections and identify important questions to be answered in the future.

KEYWORDS: Innate immunity, pattern recognition receptors, Toll-like receptors, Nod-like receptors, inflammasome, caspase-1, Interleukin-1 $\beta$, Interleukin-18, inflammation, and bacterial infection 


\section{INFLAMMASOMES: STRUCTURE AND FUNCTION}

The ability to detect infection by pathogenic microbes and to restrict their growth is fundamental for the well-being of multicellular organisms. Microbial products and endogenous "danger signals" released by infected or otherwise stressed host cells are recognized by families of pattern recognition receptors (PRR) resulting in the activation of signaling pathways that initiate the inflammatory response and regulate development of adaptive immunity. Identification and characterization of PRR has progressed very rapidly in the last few years, and their role in the innate immune response to infection continues to be unraveled. Toll-like receptor (TLR) and C-type lectins are PRR expressed on the cell surface or in endosomal compartments, while RIG-I-like receptor (RLR) are located in the cytosol (reviewed in [1]). Stimulation of these receptors results in activation of the NF- $\kappa$ B-, MAPK-, Syk-, and IRF-signaling pathways culminating in transcriptional induction and the secretion of a large number of cytokines, chemokines, and immunomodulatory factors. The Nod-like receptors (NLR) family is another group of cytoplasmic PRR that performs diverse immunological functions (reviewed in [2]). A subgroup of NLR surveys the cytoplasm for evidence of danger or infection and control activation of the inflammasome, a multiprotein complex that regulates activation of the cysteine protease caspase-1. Activation of caspase-1 in the context of the inflammasome is responsible for the proteolytic processing of the immature forms of Interleukin-1 $\beta$ (IL-1 $\beta$ ) and IL-18, two very powerful proinflammatory cytokines with pleiotropic activities [3]. While the production of most proinflammatory cytokines is primarily regulated at the transcriptional level, secretion of IL- $1 \beta$ and IL-18 requires this additional proteolytic step. Thus, IL- $1 \beta$ and IL-18 secretion is regulated in a two-step fashion. First, stimulation through TLR or RLR induces their synthesis as inactive precursors that lack signal peptide. Second, NLR-mediated inflammasome activation catalyzes the posttranslational processing that is required for their secretion and bioactivity. It should be noted that although IL- $1 \beta$ and IL-18 processing is catalyzed most efficiently by caspase-1, other proteases can process IL-1 $\beta$ under particular circumstances, like during high neutrophilic inflammation [4-6].

In addition to regulating processing and the secretion of IL- $1 \beta$ and IL-18, inflammasomes also trigger pyroptosis, a form of cell death of the infected cell that is distinct from classical apoptosis or necrosis [7]. Pyroptosis, by destroying the pool of infected cells, effectively restricts intracellular bacteria growth and dissemination and, therefore, is an efficient effector mechanism to protect the host from infection $[8,9]$.

A few inflammasomes have been recently characterized, and it is likely that many more will soon be reported. The Inflammasomes so far characterized are generally composed of a PRR, the adaptor molecule ASC, and caspase-1 (see model in Figure 1). There is evidence that other inflammatory caspases may be part of inflammasomes [10]. At least two families of PRR have been shown to form inflammasomes, the NLR and the PYHIN proteins (Pyrin and HIN200 domain-containing proteins; also known as p200 or HIN200 proteins). Members of both families have a modular domain structure. NLR are characterized by a carboxyterminal domain that contains leucine-rich repeats and that likely mediates the interaction with the ligands, a central nucleotide-binding domain that mediates oligomerization of the receptors, and an amino-terminal domain that contains either the Pyrin sequence (PYD) or the caspase activation and recruitment domain (CARD). The PYHIN family is composed of members that have varied functions [11]. PYHIN proteins are characterized by a carboxy-terminal HIN domain that is able to interact with DNA and an amino-terminal PYD domain.

Assembly of functional inflammasomes is believed to be driven by homophilic interaction between the PYD and CARD domains of the NLR/PYHIN receptors and the PYD and CARD domains of the adaptor molecule ASC and the CARD of caspase-1. Assembly of this multiprotein platform, which is reminiscent of the apoptosome, leads to activation of caspase-1 by the proximity model [12].

NLRP3 and NLRC4 are the best-characterized NLR molecules. The NLRP3 inflammasome is the most studied, and yet, the logic that oversees its activation remains elusive. NLRP3 contains a PYD domain that mediates the interaction with ASC that, acting as a bridge, recruits caspase-1. The NLRP3 inflammasome is activated by a wide variety of particles, crystals, bacterial toxins, as well as viruses bacteria, and fungi. Because of the great variability in structure and composition of the particles and stimuli 


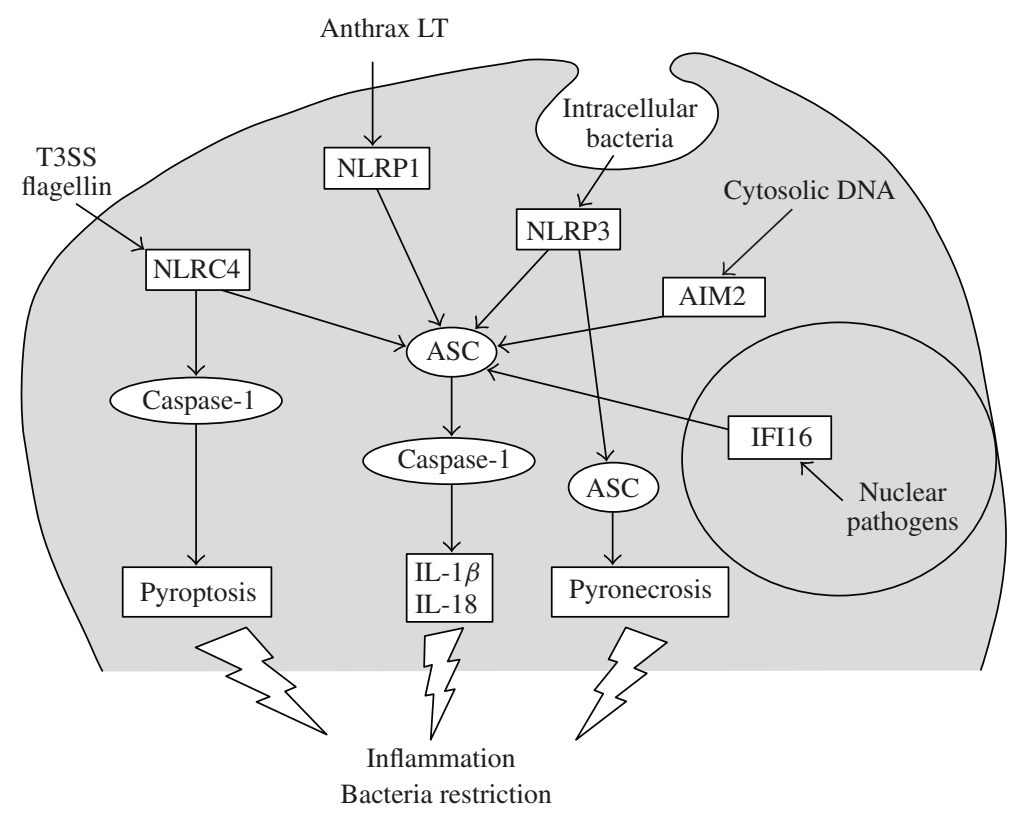

FIGURE 1

reported to activate the NLRP3 inflammasome, their direct interaction with NLRP3 seems unlikely. Rather, a more probable scenario is that NLRP3 is activated by an endogenous molecule that is generated/modified as result of the interaction of the particle/pathogen with the cell. It is possible that more than one type of NLRP3 endogenous activator exists and that different NLRP3 activators may use distinct signaling pathways to generate the same endogenous activator.

An essential requirement for NLRP3 inflammasome activation is the uptake of the particle through phagocytosis, as demonstrated by the fact that cytochalasins or other drugs that inhibit this process also prevent pro-IL-1 $\beta$ maturation in response to NLRP3-activating particles $[13,14]$. However, phagocytosis is not required for NLRP3 inflammasome activation by extracellular ATP or bacterial toxins, and conversely, phagocytosis does not always result in inflammasome activation.

One common property of the particles and agents that activate NLRP3 is their ability to induce generation of reactive oxygen species (ROS). ROS are known to regulate various signaling pathways raising the possibility of their involvement in NLRP3 activation. The majority of the evidence implicating ROS in inflammasome activation, however, was based on the use of pharmacological inhibitors, which can have unspecific effects. In fact, genetic studies using mice deficient in phagocytic oxidases have failed to confirm the importance of ROS for NLRP3 activation $[15,16]$. More recent evidence showed that the role of ROS on NLRP3 inflammasome activation was at the level of the priming step, the requirement for NF- $\kappa$ B-mediated transcriptional induction of pro-IL-1 $\beta$ and NLRP3 itself, rather than at the level of inflammasome assembly and activation of caspase-1 [17].

One of the earliest identified events triggered by all NLRP3 activator is $\mathrm{K}^{+}$efflux [18]. Prevention of $\mathrm{K}^{+}$efflux by supplementing the cell growth medium with $130 \mathrm{mM} \mathrm{KCl}$ is reported to block NLRP3 inflammasome activation in response to all known NLRP3 activators including nonparticulated ones such as extracellular ATP and the microbial toxins nigericin, maitotoxin, and gramicidin. These toxins allow fluxes of single-charged cations and cause a marked depletion of intracellular $\mathrm{K}^{+}$. An important yet unanswered question is how $\mathrm{K}^{+}$concentration regulates NLRP3 assembly into a functional inflammasome. Interestingly, $\mathrm{K}^{+}$efflux was shown to regulate also the activation of the NLRC4 and the NLRP1b inflammasomes [19-21].

One model has been proposed where lysosome destabilization would play a central role in NLRP3 activation [16]. According to this model, phagocytosis of crystals or particles that the cell is unprepared to handle properly would damage the lysosome and lead to leakage of the protease cathepsin B into the 
cytosol leading to NLRP3 activation. The idea of lysosome destabilization as an inflammasome-triggering mechanism is attractive, because it is consistent with the pathogenic mechanism of bacteria known to activate the NLRP3 inflammasome. Most of these bacteria (see below), in fact, produce a variety of toxins that open pores or damage the host cell and vacuole membrane. Activation of NLRP3 by influenza virus depends on the M2 protein, which also forms ion channels in the vacuole membrane [22]. Various crystals were also shown to cause lysosome destabilization and rupture of the lysosomal membrane. Therefore, it appears that the disruption of cell membranes integrity may be a common event triggered by the NLRP3 activators, and in this sense, the NLRP3 inflammasome may be viewed as a guardian of membrane integrity. Recent work has shown that silencing expression of the NLR molecule NLRC5 inhibits activation of the NLRP3 inflammasome by bacteria, suggesting that NLRP3 may work in concert with NLRC5 [23].

The NLRC4 inflammasome is responsive to a narrower spectrum of activators, including cytoplasmically delivered bacterial flagellin [24-26] and the basal rod constituent of various bacterial Type III secretion systems (T3SS) [27]. The T3SS apparatus and other analogous bacteria secretion systems are used by several bacteria, including those of the genus Salmonella, Listeria, Francisella, Shigella, and Legionella to inject virulence factors into the cytoplasm of target cells [28]. NLRC4 activation, at least in some experimental settings, may occur independently of ASC, presumably because the CARD domain of NLRC4 may directly interact with the CARD of caspase-1 [29-32]. Recent works [33, 34] demonstrate that the specificity of the NLRC4 inflammasome for distinct bacterial ligands is determined by its pairing with different members of the NAIP family, a subgroup of NLR molecules. Thus, NAIP5 allows the recognition of bacterial flagellin by NLRC4, while NAIP2 confers specificity for T3SS rod proteins such as Salmonella PrgJ and Burkholderia BsaK.

The form of cell death induced by the NLRC4 inflammasome is termed pyroptosis, depends on caspase-1, but occurs independently of the adaptor molecule ASC [7]. Another form of cell death, called pyronecrosis, is induced by NLRP3 and, in contrast to pyroptosis, does not require caspase-1 [9]. As the names suggest, both pyroptosis and pyronecrosis incite an inflammatory response that adds to the inflammation initiated by the recognition of microbial products. Release of the endogenous danger signal HMGB1 has been reported during pyronecrosis and pyroptosis [35].

AIM2 is a recently identified PYHIN protein that was shown to mediate innate immune responses to cytosolic DNA [36-38]. AIM2 binds cytosolic double-stranded DNA derived from a variety of pathogens including bacteria and viruses through its single HIN domain and through its PYD domain promotes the assembly of an ASC-containing inflammasome. Recently published work has identified another PYHIN family members, IFI16, that can form an inflammasome capable of activating caspase- 1 in response to viral infection [39]. Interestingly, IFI16 is reported to recognize the viral genome in the cell nucleus, the first example of a nuclear inflammasome. IFI16 is also unique in that it contains two HIN domains. One major unanswered question regarding the AIM2 inflammasome is how the bacterial DNA reaches the cytoplasm and is made available for interaction with AIM2.

\section{ROLE OF IL-1 $\beta$ AND IL-18 IN BACTERIAL INFECTIONS}

A great number of publications have documented the role of IL-18 and IL-1 $\beta$ during infections with a variety of pathogens. Almost invariably, both cytokines were found to have a protective function [40, 41].

One of the main functions of IL-18 is to promote the production of IFN $\gamma$ from T and NK cells, particularly in the presence of IL-12p70. IL-18 also promotes the secretion of other proinflammatory cytokines like TNF $\alpha$, IL- $1 \beta$, IL-8, and GM-CSF and, as a consequence, enhances expansion, migration, and activation of neutrophils during infections. In addition, IL-18 enhances cytotoxic activity and proliferation of $\mathrm{CD}^{+} \mathrm{T}$ and NK cells. The protective role of IL-18 during bacterial infections is primarily related to its ability to induce IFN $\gamma$, a cytokine that activates the microbicidal activity of macrophages through induction of nitric oxide production.

IL- $1 \beta$ has been shown to be protective in several bacterial, viral, and fungal infection models. Studies in humans have also shown that the inhibition of the function of IL-1 using the IL-1R antagonist IL-1ra 
(Kineret) is associated with increased susceptibility to bacterial infection. IL- $1 \beta$ is one of the most powerful proinflammatory cytokines; it affects virtually every organ, and several human pathologies are primarily driven by unrestrained IL- $1 \beta$ production. IL- $1 \beta$ exerts its protective action against infections by activating several responses including the rapid recruitment of neutrophils to inflammatory sites, activation of the endothelial adhesion molecules, induction of cytokines and chemokines, induction of the febrile response, and the stimulation of specific type of adaptive immunity like the Th17 response.

The inflammatory response to infection consists of several protective effector mechanisms that must be activated and orchestrated in order to maximize microbicidal functions and the stimulation of adaptive immunity while, at the same time, minimize damage to the host tissues. Alteration in this balance may result in excessive and nonresolving inflammation that is cause of severe morbidity and mortality [42]. Excessive inflammation, in fact, has been shown to be detrimental in certain infection models. For example, TLR-mediated signaling negatively affects the outcome of infections with West Nile virus [43] or influenza virus [44]. Excessive PMN recruitment is known to cause tissue damage leading to functional impairment of multiple organs, particularly the lungs [45]. Among the proinflammatory cytokines, IL- $1 \beta$ has one of the highest potential of to cause damage to the host tissues, and in fact, various mechanisms are devoted to restrain its activity, intracellularly, by carefully controlling its transcription and processing by the inflammasome and, extracellularly, by inhibition of its receptor signaling through the IL-1ra and the type II decoy receptor. Considering the potential to harm the host, it is not surprising that in some animal infection models, IL- $1 \beta$ and IL-18 were shown to be detrimental. Both cytokines were reported to have a negative effect on bacterial clearance in a model of pneumonia caused by Pseudomonas aeruginosa [46, 47]. Similarly, it has been reported that IL-18 has a detrimental role in a murine model of ehrlichiosis caused by the intracellular bacterium Ixodes ovatus ehrlichia [48]. Our recent study also showed that because of excessive recruitment of neutrophils, IL- $1 \beta$ is deleterious in a mouse model of melioidosis caused by $B$. pseudomallei (see below).

As the examples briefly mentioned in this paper will indicate, a common theme that emerges from the analysis of the literature regarding the host-pathogen interactions is the notion that in order to be effective but nonpathogenic the inflammatory response must be tailored to each specific pathogen. Thus, production of IL-18 or IL-1 $\beta$ may be protective during certain infections but deleterious for other, pyroptosis effective against some pathogens but not other.

\section{ROLE OF INFLAMMASOME IN BACTERIAL INFECTION}

Both effector mechanisms activated by the inflammasome (production of IL-1 $\beta$ and IL-18 and induction of pyroptosis and pyronecrosis) have been shown to be protective against many infectious agents. It is worth to emphasize that although inflammasome activation has been documented in response to infection with a large number of bacteria in vitro, it has proven more difficult to unequivocally determine the role of each inflammasome and effector mechanisms for the ability of animals to survive the infection. This fact likely reflects the redundancy of the pathways that lead to inflammasome activation during bacterial infection. As reviewed below, several bacteria species can activate multiple inflammasome pathways that converge on caspase- 1 activation. Thus, deficiency of caspase- 1 or IL- $1 \beta / \mathrm{IL}-18$ commonly results in a more severe phenotype than deficiency in specific NLR or PYHIN proteins. Moreover, with a few notable exceptions, it has proven difficult to determine the relative contribution of IL- $1 \beta$, IL-18, and pyroptosis to the ability to survive infection, because these responses are coregulated and, therefore, difficult to analyze separately.

\subsection{Salmonella typhimurium}

Salmonella enterica serovar Typhimurium (S.t.) is a Gram-negative bacterium that causes a systemic typhoid-like disease and serves as an experimental model for human typhoid. S.t. infects intestinal epithelial cells and macrophages and is able to survive inside the cells in a specialized vacuole. From here, the bacterium injects various virulence factors into the host cell cytoplasm using T3SS, a common strategy used 
by many bacterial pathogens. The seminal report by Mariathasan et al. [29] showed that IL-1 $\beta$ secretion and pyroptosis triggered by infection of macrophages with S.t. was dependent on NLRC4, ASC, and caspase1. Later, it was shown $[49,50]$ that NLRP3 was dispensable for those responses. Surprisingly, although $\mathrm{Casp1}^{-/-}$mice are more susceptible to infection with S.t. than WT mice, $\mathrm{Nlrp}^{-/-}, \mathrm{NlrC}^{-/-}$, or Asc ${ }^{-/-}$ mice are not $[51,52]$, suggesting that multiple pathways may lead to caspase-1 activation in response to S.t. infection. A recent paper by Broz et al. [53], in fact, showed that NLRC4 and NLRP3 are redundant for inflammasome activation during S.t. infection and that mice deficient in both NLRP3 and NLRC4 have higher bacterial burdens in organs than the single knockout mice. It is unclear whether the different bacterial load observed in that study between single and double knockout translated into significantly different survival of the infected mice. A recent paper by Miao et al. [8] employed elegant genetic manipulation to show that as S.t. strain that constitutively expresses flagellin has attenuated virulence. In the early phase of the infection, S.t. relies on the SPI1 T3SS to infect intestinal epithelial cells and inject virulence factors, including flagellin and the PrgJ rod protein, both of which are detected by the NLRC4 inflammasome. However, during infection of macrophages and systemic dissemination, S.t. manages to elude inflammasome detection by silencing SPI1 and turning on SPI2, a different T3SS that does not activate the inflammasome. The increased clearance of the flagellin-expressing transgenic S.t. was due to the activation of the NLRC4 inflammasome and induction of pyroptosis but was independent of the production of IL- $1 \beta$ and IL-18. This is one of the first reports to convincingly show the importance and effectiveness of pyroptosis for restriction of bacteria in vivo. This study also indicates that evasion of NLRC4-mediated detection can be a very powerful virulence strategy.

\subsection{Listeria monocytogenes}

Listeria monocytogenes (L.m.) is an intracellular, Gram-positive bacterium that can cause listeriosis, a potentially lethal food-borne infection. L.m. infects many cell type including intestinal epithelial cells and macrophages. The phagocytosed bacterium produces the listeriolysin $\mathrm{O}$ toxin that breaches the vacuolar membrane allowing the bacterium to escape the phagosome and colonize the cytoplasm. Caspase- 1 activation and IL-1 $\beta$ secretion by macrophages infected with L.m. was first reported to be dependent on NLRP3 and ASC [49]. More recently, several groups demonstrated that the NLRP3, NLRC4, and AIM2 inflammasomes contribute to caspase- 1 activation, pyroptosis, and the secretion of IL- $1 \beta$ and IL-18 during L.m. infection [54-57]. Thus, as is the case for other intracellular bacteria, different stages of the infection process can be detected by distinct inflammasomes. This level of redundancy explains the observation that Casp1 $^{-/-}$mice are more susceptible to infection than WT mice, yet no significant difference in animal survival has been reported for mice deficient in the other inflammasome components NLRP3, ASC, NLRC4, and AIM2. IL-18 has been shown to be protective during infection with L.m. [58], while IL-1 $\beta$, although protective in some studies, seems to play a less important role [59].

\subsection{Legionella pneumophila}

Legionella pneumophila (L.p.) is a motile Gram-negative bacterium that causes the Legionnaires' disease, a severe form of pneumonia. L.p. infects macrophages and is able to survive inside an intracellular specialized vacuole. Through a Type IV secretion apparatus, L.p. injects virulence factors into the host cytoplasm. Infection of macrophages with L.p. induces caspase- 1 activation and the release of IL-1 $\beta$, IL-18, and pyroptosis [26, 32, 60-62]. Inflammasome activation requires the Type IV secretion system and the flagellin and is mediated by NLRC4. The adaptor ASC is required for IL- $1 \beta$ and IL-18 secretion but not pyroptosis. Nlrc4 ${ }^{-/-}$, Casp1 $1^{-/-}$, or $\mathrm{Il}-1 b^{-/-} / \mathrm{Il}-18^{-/-}$mice infected with L.p. have higher bacterial burdens than WT mice, while ASC seems to be dispensable for bacteria restriction in vivo [32, 62]. Flagellin-deficient L.p. is more virulent than the WT bacterium [61, 62], confirming the effectiveness of inflammasomemediated responses at restricting bacteria growth. It has been demonstrated that activation of the NLRC4 inflammasome by Legionella flagellin is also dependent on the Naip5 molecule, an NLR member that may directly interact with NLRC4 [60, 61, 63]. 


\subsection{Shigella flexneri}

The Gram-negative bacteria Shigella flexneri (S.f.) is the causative agent of bacillary dysentery. Activation of caspase- 1 and IL- $1 \beta$ secretion by macrophages infected with S.f. are mediated by the NLRC4 inflammasome [30]. Although S.f. does not possess a flagellum, it has been showed that the basal rod proteins that constitute the T3SS of this bacterium can be detected by the NLRC4 inflammasome [27]. S.f. infection of macrophages also triggers cell death by pyronecrosis in a NLRP3- and ASC-dependent but caspase-1-independent fashion [9]. Caspase-1- and IL-18-deficient mice are more susceptible to S.f. infection, while $I l-1 b^{-/-}$have susceptibility similar to WT mice [64].

\subsection{Pseudomonas aeruginosa}

Pseudomonas aeruginosa (P.a.) is a Gram-negative flagellate bacterium that causes opportunistic infections in immunocompromised individuals. P.a. employs different T3SS to inject virulence factors into the cytoplasm of host cells. Caspase- 1 activation, IL- $1 \beta$ secretion, and pyroptosis during P.a. infection of macrophages were shown to be dependent on the NLRC4 inflammasome [65, 66]. P.a. flagellin transfected into the cytoplasm was shown to activate the NLRC4 inflammasome [31] though P.a. flagellin mutants were also able to activate the NLRC4 inflammasome. This is consistent with the demonstrated ability of NLRC4 to recognize the basal rod components of the P.a.T3SS [27]. Nlrc $4^{-/-}$mice infected with P.a. had higher bacterial burdens than WT mice though the survival was not different [31]. In contrast, it was demonstrated that IL- $1 \beta$ and IL-18 had a negative effect on bacterial clearance in a model of pneumonia caused by P.a. $[46,47]$. These results, thus, would suggest that the protective role of the inflammasome during pneumonia caused by $P . a$. is mediated by pyroptosis and is independent of production of IL- $1 \beta$ and IL-18, a scenario that is reminiscent of our observation regarding the infection with Burkholderia pseudomallei bacterium (see below).

\subsection{Francisella tularensis}

Francisella tularensis (F.t.) is a Gram-negative bacterium that causes tularemia. F.t. infects macrophages and epithelial cells and is able to evade the phagosome and invade and replicate in the host cell cytoplasm. F.t. infection of macrophages leads to caspase- 1 activation, pyroptosis, and the secretion of IL- $1 \beta$ and IL18 [67]. These responses were reported to require phagosome escape and ASC but not NLRC4 or NLRP3. Recent works $[54,68,69]$ have shown that inflammasome activation by F.t. is mediated by the AIM2 inflammasome that detects F.t. DNA in the cytoplasm. The role of IL-1 $\beta$ and IL-18 in the control of F.t. infection in vivo has not been examined in detail. Administration of IL- $1 \beta$ and IL-18 neutralizing antibodies increased bacterial burdens in organs though not as much as absence of caspase-1 [67]. Our unpublished work indicates that IL-18 plays a prominent role in the early phase of the infection, while the role of IL- $1 \beta$ becomes more important in the later stages of the infection possibly because of the requirement of IL- $1 \beta$ for development of adaptive immunity against F.t.

\subsection{Burkholderia pseudomallei}

Burkholderia pseudomallei (B.p.), the etiologic agent of melioidosis, is a Gram-negative flagellated bacterium that infects macrophages and other cell types. B.p. infection was shown to result in caspase-1 activation, pyroptosis, and the secretion of IL-1 $\beta$ and IL-18 [70]. We have recently shown (Ceballos-Olvera et al., manuscript submitted) that NLRP3 and NLRC4 differentially regulate pyroptosis and production of IL- $1 \beta$ and IL-18 and are critical for resistance to melioidosis. In vitro production of IL- $1 \beta$ and IL-18 by BMDC infected with B.p. was dependent on NLRC4 and NLRP3, while pyroptosis required only NLRC4. Mice deficient in the inflammasome components ASC, caspase-1, NLRC4, and NLRP3 were dramatically more susceptible to lung infection with B.p. than WT mice. The heightened susceptibility of Nlrp $3^{-/-}$mice was due to decreased production of IL-18 and IL-1 $\beta$. In contrast, the high susceptibility of Nlrc $4^{-/-}$mice 
was not due to lack of IL- $1 \beta$ and IL-18, which, in fact, were produced in higher amount than WT mice, but rather to decreased pyroptosis and consequently higher bacterial burdens. Analyses of IL-18-deficient mice infected with B.p. revealed that IL-18 is essential for survival primarily because of its ability to induce IFN $\gamma$ production. In contrast, studies using IL-1RI-deficient mice or WT mice treated with either IL-1 $\beta$ or IL-1 receptor agonist revealed that IL- $1 \beta$ can have deleterious effects during melioidosis. The detrimental role of IL- $1 \beta$ appeared to be due, in part, to excessive recruitment to the lung of neutrophils, cells that are permissive to B.p. intracellular growth, because they do not express NLRC4 and, therefore, fail to undergo pyroptosis. Thus, the NLRP3 and NLRC4 inflammasomes have nonredundant protective roles in melioidosis: NLRC4 regulates pyroptosis, while NLRP3 regulates production of protective IL-18 and deleterious IL- $1 \beta$.

\subsection{Mycobacteruim tubeculosis}

Secretion IL-1 $\beta$ and IL-18 by macrophages infected with Mycobacteruim tubeculosis (Mtb) was reported to be dependent on NLRP3 and ASC but not NLRC4 [71]. The mycobacterial secretion system ESX-1 was required for the inflammasome activation [72]. $\mathrm{Il}-1 \mathrm{rl} \mathrm{I}^{-/-}$and $\mathrm{Il}-18^{-/-}$mice are very susceptible to $\mathrm{Mtb}$ infection [73-75]. $\mathrm{Casp1}^{-/-}$and $A s \mathrm{c}^{-/-}$mice are also more susceptible than WT mice due to defective granuloma formation [76]. However, the resistance of $N l r p 3^{-/-}$mice to $M t b$ infection is not significantly different from that of WT mice, suggesting the existence of other pathways for inflammasome activation during $M t b$ infection. Surprisingly, the production of IL-1 $\beta$ during $M t b$ infection was reported to occur also in a caspase-1-independent fashion [77].

\subsection{Staphylococcus aureus}

Activation of the inflammasome by Staphylococcus aureus (S.a.) was reported to be dependent on NLRP3 and ASC [78, 79]. Inflammasome activation by S.a. required the alpha, beta, and gamma hemolysins [78, 79]. It has been shown that phagocytosis of the particulate peptidoglycan from the bacterial cell wall can trigger secretion of IL-1 $\beta$. Interestingly, S.a. can modify its cell wall peptidoglycan to avoid recognition by NLRP3 inflammasome [80]. IL-1 $\beta$ production is important for neutrophil recruitment and bacterial clearance during S.a. infection in vivo [81].

\subsection{Streptococcus Group}

Activation of NLRP3 inflammasome and IL-1 $\beta$ secretion by Streptococcus pneumoniae (S.p.) requires the bacterial toxin pneumolysin [82]. IL-18 was shown to provide protection from S. pneumoniae and Group B Streptococci infection through its ability to induce IFN $\gamma$. [83, 84]. Similarly to S.p., S. pyogenes produces a pore-forming toxin, streptolysin $\mathrm{O}$, which activates caspase-1 via NLRP3 inflammasome, and ASC resulting in secretion of IL-1 $\beta$. [85]. In addition, it has also been reported that NLRC4 senses Group A Streptococci associate with Beclin1 and negatively regulates maturation of autophagosome and endosome [86].

\subsection{Bacillus anthracis}

The lethal toxin (LT) of $B$. anthracis was shown to activate the NLRP1 inflammasome in mouse macrophages resulting in IL-1 $\beta$ secretion [87-89]. Anthrax spores were also shown to induce pyroptosis of infected macrophages, which was originally proposed to be a protective mechanism in an in vivo infection model [89]. Activation of the NLRP1b inflammasome by LT involves lysosomal membrane permeabilization and may lead to cathepsin B release into in the cytosol [90], suggesting that activation of the NLRP1 and NLRP3 inflammasomes may share common mechanistic aspects.

\subsection{Other Bacteria}

Several other bacteria have been shown to activate the NLRP3 inflammasome resulting in IL- $1 \beta$ secretion and caspase-1-dependent pyroptosis or caspase-1-independent pyronecrosis. In several cases, the 
inflammasome activation is due to the action of toxins that disrupt cell membrane such as the aerolysin of Aeromonas hydrophila [91] or the hemolysis of Vibrio species [92]. Infection with Neisseria gonorea [93] and Klebsiella pleumoniae [94] activates NLRP3 and induces pyronecrosis and release of HMGB1. Chlamyidia species that reside in a modified vacuole inside the cell also activate NLRP3 [95, 96]. Activation of both NLRC4 and NLRP3 inflammasomes by Yersinia pestis depends on the T3SS and is antagonized by YopK, a bacterial virulence factor that is injected into the host cell cytoplasm [97].

\section{CONCLUSIONS}

The discovery of the inflammasome and the PRR that constitutes it has spurred a resurgence in the interest in "old" cytokines like IL-1 $\beta$ and IL-18 as well as interests into new forms of cell death. The challenge for the future will be to determine the role played by each inflammasome effector mechanisms in the pathogenesis of infectious diseases and other inflammatory pathologies and to conceive novel clinical interventions to selectively block the deleterious responses and boost the protective ones.

\section{ACKNOWLEDGMENT}

This work was supported by National Institute of Health Grants no. AI076835 and AI081861-01 (F.Re.).

\section{REFERENCES}

[1] H. Kumar, T. Kawai, and S. Akira, "Pathogen recognition by the innate immune system," International Reviews of Immunology, vol. 30, no. 1, pp. 16-34, 2011.

[2] B. K. Davis, H. Wen, and J. P. Ting, "The inflammasome NLRs in immunity, inflammation, and associated diseases," Annual Review of Immunology, vol. 29, pp. 707-735, 2011.

[3] C. A. Dinarello, "Interleukin 1 and interleukin 18 as mediators of inflammation and the aging process," American Journal of Clinical Nutrition, vol. 83, no. 2, pp. S447-S455, 2006.

[4] G. Fantuzzi, G. Ku, M. W. Harding et al., "Response to local inflammation of IL-1 $\beta$-converting enzyme-deficient mice," Journal of Immunology, vol. 158, no. 4, pp. 1818-1824, 1997.

[5] C. Coeshott, C. Ohnemus, A. Pilyavskaya et al., "Converting enzyme-independent release of tumor necrosis factor $\alpha$ and IL-1 $\beta$ from a stimulated human monocytic cell line in the presence of activated neutrophils or purified proteinase 3," Proceedings of the National Academy of Sciences of the United States of America, vol. 96, no. 11, pp. 6261-6266, 1999.

[6] M. Guma, L. Ronacher, R. Liu-Bryan, S. Takai, M. Karin, and M. Corr, "Caspase 1-independent activation of interleukin- $1 \beta$ in neutrophil-predominant inflammation," Arthritis and Rheumatism, vol. 60, no. 12, pp. 36423650, 2009.

[7] I. E. Brodsky and R. Medzhitov, "Pyroptosis: macrophage suicide exposes hidden invaders," Current Biology, vol. 21, no. 2, pp. R72-R75, 2011.

[8] E. A. Miao, I. A. Leaf, P. M. Treuting et al., "Caspase-1-induced pyroptosis is an innate immune effector mechanism against intracellular bacteria," Nature Immunology, vol. 11, no. 12, pp. 1136-1142, 2010.

[9] S. B. Willingham, D. T. Bergstralh, W. O'Connor et al., "Microbial pathogen-induced necrotic cell death mediated by the inflammasome components CIAS1/Cryopyrin/NLRP3 and ASC," Cell Host and Microbe, vol. 2, no. 3, pp. 147-159, 2007.

[10] F. Martinon and J. Tschopp, "Inflammatory caspases: linking an intracellular innate immune system to autoinflammatory diseases," Cell, vol. 117, no. 5, pp. 561-574, 2004.

[11] D. Goubau, J. Rehwinkel, and R. E. Sousa, "PYHIN proteins: center stage in DNA sensing," Nature Immunology, vol. 11, no. 11, pp. 984-986, 2010.

[12] G. S. Salvesen and V. M. Dixit, "Caspase activation: the induced-proximity model," Proceedings of the National Academy of Sciences of the United States of America, vol. 96, no. 20, pp. 10964-10967, 1999.

[13] F. Martinon, V. Petrilli, A. Mayor, A. Tardivel, and J. Tschopp, "Gout-associated uric acid crystals activate the NALP3 inflammasome," Nature, vol. 440, no. 7081, pp. 237-241, 2006. 
[14] H. Li, S. Nookala, and F. Re, "Aluminum hydroxide adjuvants activate caspase-1 and induce IL-1 $\beta$ and IL-18 release," Journal of Immunology, vol. 178, no. 8, pp. 5271-5276, 2007.

[15] C. Dostert, G. Guarda, J. F. Romero et al., "Malarial hemozoin is a Nalp3 inflammasome activating danger signal," Plos One, vol. 4, no. 8, Article ID e6510, 2009.

[16] V. Hornung, F. Bauernfeind, A. Halle et al., "Silica crystals and aluminum salts activate the NALP3 inflammasome through phagosomal destabilization," Nature Immunology, vol. 9, no. 8, pp. 847-856, 2008.

[17] F. Bauernfeind, E. Bartok, A. Rieger, L. Franchi, G. Nunez, and V. Hornung, "Cutting edge: reactive oxygen species inhibitors block priming, but not activation, of the NLRP3 inflammasome," Journal of Immunology, vol. 187, no. 2, pp. 613-617, 2011.

[18] F. Martinon, K. Burns, and J. Tschopp, "The inflammasome: a molecular platform triggering activation of inflammatory caspases and processing of proIL- $\beta$," Molecular Cell, vol. 10, no. 2, pp. 417-426, 2002.

[19] C. S. Arlehamn, V. Petrilli, O. Gross, J. Tschopp, and T. J. Evans, "The role of potassium in inflammasome activation by bacteria," Journal of Biological Chemistry, vol. 285, no. 14, pp. 10508-10518, 2010.

[20] S. L. Fink, T. Bergsbaken, and B. T. Cookson, "Anthrax lethal toxin and Salmonella elicit the common cell death pathway of caspase-1-dependent pyroptosis via distinct mechanisms," Proceedings of the National Academy of Sciences of the United States of America, vol. 105, no. 11, pp. 4312-4317, 2008.

[21] K. E. Wickliffe, S. H. Leppla, and M. Moayeri, "Anthrax lethal toxin-induced inflammasome formation and caspase-1 activation are late events dependent on ion fluxes and the proteasome," Cellular Microbiology, vol. 10, no. 2, pp. 332-343, 2008.

[22] T. Ichinohe, I. K. Pang, and A. Iwasaki, "Influenza virus activates inflammasomes via its intracellular M2 ion channel," Nature Immunology, vol. 11, no. 5, pp. 404-410, 2010.

[23] B. K. Davis, R. A. Roberts, M. T. Huang et al., "Cutting edge: NLRC5-dependent activation of the inflammasome," Journal of Immunology, vol. 186, no. 3, pp. 1333-1337, 2011.

[24] L. Franchi, A. Amer, M. Body-Malapel et al., "Cytosolic flagellin requires Ipaf for activation of caspase-1 and interleukin $1 \beta$ in salmonella-infected macrophages," Nature Immunology, vol. 7, no. 6, pp. 576-582, 2006.

[25] E. A. Miao, C. M. Alpuche-Aranda, M. Dors et al., "Cytoplasmic flagellin activates caspase-1 and secretion of interleukin $1 \beta$ via Ipaf,” Nature Immunology, vol. 7, no. 6, pp. 569-575, 2006.

[26] A. B. Molofsky, B. G. Byrne, N. N. Whitfield et al., "Cytosolic recognition of flagellin by mouse macrophages restricts Legionella pneumophila infection," Journal of Experimental Medicine, vol. 203, no. 4, pp. 1093-1104, 2006.

[27] E. A. Miao, D. P. Mao, N. Yudkovsky et al., "Innate immune detection of the type III secretion apparatus through the NLRC4 inflammasome," Proceedings of the National Academy of Sciences of the United States of America, vol. 107, no. 7, pp. 3076-3080, 2010.

[28] C. J. Hueck, "Type III protein secretion systems in bacterial pathogens of animals and plants," Microbiology and Molecular Biology Reviews, vol. 62, no. 2, pp. 379-433, 1998.

[29] S. Mariathasan, K. Hewton, D. M. Monack et al., "Differential activation of the inflammasome by caspase-1 adaptors ASC and Ipaf," Nature, vol. 430, no. 6996, pp. 213-218, 2004.

[30] T. Suzuki, L. Franchi, C. Toma et al., "Differential regulation of caspase-1 activation, pyroptosis, and autophagy via Ipaf and ASC in Shigella-infected macrophages," Plos Pathogens, vol. 3, no. 8, Article ID e111, 2007.

[31] L. Franchi, J. Stoolman, T. D. Kanneganti, A. Verma, R. Ramphal, and G. Núñez, "Critical role for Ipaf in Pseudomonas aeruginosa-induced caspase-1 activation," European Journal of Immunology, vol. 37, no. 11, pp. 3030-3039, 2007.

[32] C. L. Case, S. Shin, and C. R. Roy, "Asc and Ipaf inflammasomes direct distinct pathways for caspase-1 activation in response to Legionella pneumophila," Infection and Immunity, vol. 77, no. 5, pp. 1981-1991, 2009.

[33] E. M. Kofoed and R. E. Vance, "Innate immune recognition of bacterial ligands by NAIPs determines inflammasome specificity," Nature, vol. 477, no. 7366, pp. 592-595, 2011.

[34] Y. Zhao, J. Yang, J. Shi et al., "The NLRC4 inflammasome receptors for bacterial flagellin and type III secretion apparatus," Nature, vol. 477, no. 7366, pp. 596-600, 2011.

[35] L. van de Walle, T. D. Kanneganti, and M. Lamkanfi, "HMGB1 release by inflammasomes," Virulence, vol. 2, no. 2, pp. 162-165, 2011.

[36] V. Hornung, A. Ablasser, M. Charrel-Dennis et al., "AIM2 recognizes cytosolic dsDNA and forms a caspase-1activating inflammasome with ASC," Nature, vol. 458, no. 7237, pp. 514-518, 2009. 
[37] T. Fernandes-Alnemri, J. W. Yu, P. Datta, J. Wu, and E. S. Alnemri, "AIM2 activates the inflammasome and cell death in response to cytoplasmic DNA," Nature, vol. 458, no. 7237, pp. 509-513, 2009.

[38] K. Schroder, D. A. Muruve, and J. Tschopp, "Innate immunity: cytoplasmic DNA sensing by the AIM2 inflammasome," Current Biology, vol. 19, no. 6, pp. R262-R265, 2009.

[39] N. Kerur, M. V. Veettil, N. Sharma-Walia et al., "IFI16 acts as a nuclear pathogen sensor to induce the inflammasome in response to Kaposi Sarcoma-associated herpesvirus infection," Cell Host and Microbe, vol. 9, no. 5, pp. 363-375, 2011.

[40] C. A. Dinarello and G. Fantuzzi, "Interleukin-18 and host defense against infection," Journal of Infectious Diseases, vol. 187, supplement 2, pp. S370-S384, 2003.

[41] C. A. Dinarello, "Immunological and inflammatory functions of the interleukin-1 family," Annual Review of Immunology, vol. 27, pp. 519-550, 2009.

[42] C. Nathan and A. Ding, "Nonresolving inflammation," Cell, vol. 140, no. 6, pp. 871-882, 2010.

[43] T. Wang, T. Town, L. Alexopoulou, J. F. Anderson, E. Fikrig, and R. A. Flavell, "Toll-like receptor 3 mediates West Nile virus entry into the brain causing lethal encephalitis," Nature Medicine, vol. 10, no. 12, pp. 1366-1373, 2004.

[44] R. Le Goffic, V. Balloy, M. Lagranderie et al., "Detrimental contribution of the Toll-like receptor (TLR)3 to influenza A virus-induced acute pneumonia," Plos Pathogens, vol. 2, no. 6, e53 pages, 2006.

[45] R. L. Zemans, S. P. Colgan, and G. P. Downey, "Transepithelial migration of neutrophils: mechanisms and implications for acute lung injury," American Journal of Respiratory Cell and Molecular Biology, vol. 40, no. 5, pp. 519-535, 2009.

[46] M. J. Schultz, S. Knapp, S. Florquin et al., "Interleukin-18 impairs the pulmonary host response to Pseudomonas aeruginosa," Infection and Immunity, vol. 71, no. 4, pp. 1630-1634, 2003.

[47] M. J. Schultz, A. W. Rijneveld, S. Florquin, C. K. Edwards, C. A. Dinarello, and T. van der Poll, "Role of interleukin-1 in the pulmonary immune response during Pseudomonas aeruginosa pneumonia," American Journal of Physiology, vol. 282, no. 2, pp. L285-L290, 2002.

[48] P. Ghose, A. Q. Ali, R. Fang, D. Forbes, B. Ballard, and N. Ismail, "The interaction between IL-18 and IL-18 receptor limits the magnitude of protective immunity and enhances pathogenic responses following infection with intracellular bacteria," Journal of Immunology, vol. 187, no. 3, pp. 1333-1346, 2011.

[49] S. Mariathasan, D. S. Weiss, K. Newton et al., "Cryopyrin activates the inflammasome in response to toxins and ATP," Nature, vol. 440, no. 7081, pp. 228-232, 2006.

[50] F. S. Sutterwala, Y. Ogura, M. Szczepanik et al., "Critical role for NALP3/CIAS1/cryopyrin in innate and adaptive immunity through its regulation of caspase-1," Immunity, vol. 24, no. 3, pp. 317-327, 2006.

[51] B. Raupach, S. K. Peuschel, D. M. Monack, and A. Zychlinsky, "Caspase-1-mediated activation of interleukin$1 \beta$ (IL-1 $\beta$ ) and IL-18 contributes to innate immune defenses against Salmonella enterica serovar Typhimurium infection," Infection and Immunity, vol. 74, no. 8, pp. 4922-4926, 2006.

[52] M. Lara-Tejero, F. S. Sutterwala, Y. Ogura et al., "Role of the caspase-1 inflammasome in Salmonella typhimurium pathogenesis," Journal of Experimental Medicine, vol. 203, no. 6, pp. 1407-1412, 2006.

[53] P. Broz, K. Newton, M. Lamkanfi, S. Mariathasan, V. M. Dixit, and D. M. Monack, "Redundant roles for inflammasome receptors NLRP3 and NLRC4 in host defense against Salmonella," Journal of Experimental Medicine, vol. 207, no. 8, pp. 1745-1755, 2010.

[54] V. A. Rathinam, Z. Jiang, S. N. Waggoner et al., "The AIM2 inflammasome is essential for host defense against cytosolic bacteria and DNA viruses," Nature Immunology, vol. 11, no. 5, pp. 395-402, 2010.

[55] S. Kim, F. Bauernfeind, A. Ablasser et al., "Listeria monocytogenes is sensed by the NLRP3 and AIM2 inflammasome," European Journal of Immunology, vol. 40, no. 6, pp. 1545-1551, 2010.

[56] J. Wu, T. Fernandes-Alnemri, and E. S. Alnemri, "Involvement of the AIM2, NLRC4, and NLRP3 inflammasomes in caspase-1 activation by Listeria monocytogenes," Journal of Clinical Immunology, vol. 30, no. 5, pp. 693-702, 2010.

[57] S. E. Warren, A. Armstrong, M. K. Hamilton et al., "Cutting edge: cytosolic bacterial DNA activates the inflammasome via Aim2," Journal of Immunology, vol. 185, no. 2, pp. 818-821, 2010. 
[58] N. M. Tsuji, H. Tsutsui, E. Seki et al., "Roles of caspase-1 in Listeria infection in mice," International Immunology, vol. 16, no. 2, pp. 335-343, 2004.

[59] H. Zheng, D. Fletcher, W. Kozak et al., "Resistance to fever induction and impaired acute-phase response in interleukin-1 $\beta$-deficient mice," Immunity, vol. 3, no. 1, pp. 9-19, 1995.

[60] D. S. Zamboni, K. S. Kobayashi, T. Kohlsdorf et al., "The Bircle cytosolic pattern-recognition receptor contributes to the detection and control of Legionella pneumophila infection," Nature Immunology, vol. 7, no. 3, pp. 318-325, 2006.

[61] T. Ren, D. S. Zamboni, C. R. Roy, W. F. Dietrich, and R. E. Vance, "Flagellin-deficient Legionella mutants evade caspase-1- and Naip5-mediated macrophage immunity," Plos Pathogens, vol. 2, no. 3, e18 pages, 2006.

[62] A. Amer, L. Franchi, T. D. Kanneganti et al., "Regulation of Legionella phagosome maturation and infection through flagellin and host Ipaf," Journal of Biological Chemistry, vol. 281, no. 46, pp. 35217-35223, 2006.

[63] K. L. Lightfield, J. Persson, N. J. Trinidad et al., "Differential requirements for NAIP5 in activation of the NLRC4 inflammasome," Infection and Immunity, vol. 79, no. 4, pp. 1606-1614, 2011.

[64] P. J. Sansonetti, A. Phalipon, J. Arondel et al., "Caspase-1 activation of IL-1 $\beta$ and IL-18 are essential for Shigella flexneri-induced inflammation," Immunity, vol. 12, no. 5, pp. 581-590, 2000.

[65] E. A. Miao, R. K. Ernst, M. Dors, D. P. Mao, and A. Aderem, "Pseudomonas aeruginosa activates caspase 1 through Ipaf," Proceedings of the National Academy of Sciences of the United States of America, vol. 105, no. 7, pp. 2562-2567, 2008.

[66] F. S. Sutterwala, L. A. Mijares, L. Li, Y. Ogura, B. I. Kazmierczak, and R. A. Flavell, "Immune recognition of Pseudomonas aeruginosa mediated by the IPAF/NLRC4 inflammasome," Journal of Experimental Medicine, vol. 204, no. 13, pp. 3235-3245, 2007.

[67] S. Mariathasan, D. S. Weiss, V. M. Dixit, and D. M. Monack, "Innate immunity against Francisella tularensis is dependent on the ASC/caspase-1 axis," Journal of Experimental Medicine, vol. 202, no. 8, pp. 1043-1049, 2005.

[68] T. Fernandes-Alnemri, J. W. Yu, C. Juliana et al., "The AIM2 inflammasome is critical for innate immunity to Francisella tularensis," Nature Immunology, vol. 11, no. 5, pp. 385-393, 2010.

[69] J. W. Jones, N. Kayagaki, P. Broz et al., "Absent in melanoma 2 is required for innate immune recognition of Francisella tularensis," Proceedings of the National Academy of Sciences of the United States of America, vol. 107, no. 21, pp. 9771-9776, 2010.

[70] K. Breitbach, G. W. Sun, J. Kohler et al., "Caspase-1 mediates resistance in murine melioidosis," Infection and Immunity, vol. 77, no. 4, pp. 1589-1595, 2009.

[71] I. C. Koo, C. Wang, S. Raghavan, J. H. Morisaki, J. S. Cox, and E. J. Brown, "ESX-1-dependent cytolysis in lysosome secretion and inflammasome activation during mycobacterial infection," Cellular Microbiology, vol. 10, no. 9, pp. 1866-1878, 2008.

[72] B. B. Mishra, P. Moura-Alves, A. Sonawane et al., "Mycobacterium tuberculosis protein ESAT-6 is a potent activator of the NLRP3/ASC inflammasome," Cellular Microbiology, vol. 12, no. 8, pp. 1046-1063, 2010.

[73] I. Sugawara, H. Yamada, H. Kaneko, S. Mizuno, K. Takeda, and S. Akira, "Role of interleukin-18 (IL-18) in mycobacterial infection in IL-18- gene-disrupted mice," Infection and Immunity, vol. 67, no. 5, pp. 2585-2589, 1999.

[74] I. Sugawara, H. Yamada, S. Hua, and S. Mizuno, "Role of interleukin (IL)-1 type 1 receptor in mycobacterial infection," Microbiology and Immunology, vol. 45, no. 11, pp. 743-750, 2001.

[75] N. P. Juffermans, S. Florquin, L. Camoglio et al., "Interleukin-1 signaling is essential for host defense during murine pulmonary tuberculosis," Journal of Infectious Diseases, vol. 182, no. 3, pp. 902-908, 2000.

[76] E. McElvania Tekippe, I. C. Allen, P. D. Hulseberg et al., "Granuloma formation and host defense in chronic Mycobacterium tuberculosis infection requires PYCARD/ASC but not NLRP3 or caspase-1," Plos One, vol. 5, no. 8, Article ID e12320, 2010.

[77] K. D. Mayer-Barber, D. L. Barber, K. Shenderov et al., "Cutting edge: caspase-1 independent IL-1 $\beta$ production is critical for host resistance to mycobacterium tuberculosis and does not require TLR signaling in vivo," Journal of Immunology, vol. 184, no. 7, pp. 3326-3330, 2010.

[78] R. Munoz-Planillo, L. Franchi, L. S. Miller, and G. Nunez, "A critical role for hemolysins and bacterial lipoproteins in Staphylococcus aureus-induced activation of the Nlrp3 inflammasome," Journal of Immunology, vol. 183, no. 6, pp. 3942-3948, 2009. 
[79] R. R. Craven, X. Gao, I. C. Allen et al., "Staphylococcus aureus $\alpha$-hemolysin activates the NLRP3-inflammasome in human and mouse monocytic cells," Plos One, vol. 4, no. 10, Article ID e7446, 2009.

[80] T. Shimada, B. G. Park, A. J. Wolf et al., "Staphylococcus aureus evades lysozyme-based peptidoglycan digestion that links phagocytosis, inflammasome activation, and IL-1 $\beta$ secretion," Cell Host and Microbe, vol. 7, no. 1, pp. 38-49, 2010.

[81] L. S. Miller, E. M. Pietras, L. H. Uricchio et al., "Inflammasome-mediated production of IL-1 $\beta$ is required for neutrophil recruitment against Staphylococcus aureus in vivo," Journal of Immunology, vol. 179, no. 10, pp. 6933-6942, 2007.

[82] E. A. McNeela, A. Burke, D. R. Neill et al., "Pneumolysin activates the NLRP3 inflammasome and promotes proinflammatory cytokines independently of TLR4," Plos Pathogens, vol. 6, no. 11, Article ID e1001191, 2010.

[83] F. N. Lauw, J. Branger, S. Florquin et al., "IL-18 improves the early antimicrobial host response to pneumococcal pneumonia," Journal of Immunology, vol. 168, no. 1, pp. 372-378, 2002.

[84] V. Cusumano, A. Midiri, V. V. Cusumano et al., "Interleukin-18 is an essential element in host resistance to experimental group B streptococcal disease in neonates," Infection and Immunity, vol. 72, no. 1, pp. 295-300, 2004.

[85] J. Harder, L. Franchi, R. Munoz-Planillo, J. H. Park, T. Reimer, and G. Nunez, "Activation of the Nlrp3 inflammasome by Streptococcus pyogenes requires streptolysin $\mathrm{O}$ and NF- $\kappa \mathrm{B}$ activation but proceeds independently of TLR signaling and P2X7 receptor," Journal of Immunology, vol. 183, no. 9, pp. 5823-5829, 2009.

[86] N. Jounai, K. Kobiyama, M. Shiina, K. Ogata, K. J. Ishii, and F. Takeshita, "NLRP4 negatively regulates autophagic processes through an association with Beclin1," Journal of Immunology, vol. 186, no. 3, pp. 16461655, 2011.

[87] E. D. Boyden and W. F. Dietrich, "Nalp1b controls mouse macrophage susceptibility to anthrax lethal toxin," Nature Genetics, vol. 38, no. 2, pp. 240-244, 2006.

[88] M. Moayeri, D. Crown, Z. L. Newman et al., "Inflammasome sensor Nlrp1b-dependent resistance to anthrax is mediated by caspase-1, IL-1 signaling and neutrophil recruitment," Plos Pathogens, vol. 6, no. 12, Article ID e1001222, 2010.

[89] J. K. Terra, C. K. Cote, B. France et al., "Cutting edge: resistance to Bacillus anthracis infection mediated by a lethal toxin sensitive allele of Nalp1b/Nlrp1b," Journal of Immunology, vol. 184, no. 1, pp. 17-20, 2010.

[90] K. M. Averette, M. R. Pratt, Y. Yang et al., "Anthrax lethal toxin induced lysosomal membrane permeabilization and cytosolic cathepsin release is Nlrp1b/Nalp1b-dependent," Plos One, vol. 4, no. 11, Article ID e7913, 2009.

[91] A. J. McCoy, Y. Koizumi, N. Higa, and T. Suzuki, "Differential regulation of caspase-1 activation via NLRP3/NLRC4 inflammasomes mediated by aerolysin and type III secretion system during Aeromonas veronii infection," Journal of Immunology, vol. 185, no. 11, pp. 7077-7084, 2010.

[92] C. Toma, N. Higa, Y. Koizumi et al., "Pathogenic Vibrio activate NLRP3 inflammasome via cytotoxins and TLR/nucleotide-binding oligomerization domain-mediated NF- $\kappa$ B signaling," Journal of Immunology, vol. 184, no. 9, pp. 5287-5297, 2010.

[93] J. A. Duncan, X. Gao, M. T. Huang et al., "Neisseria gonorrhoeae activates the proteinase cathepsin B to mediate the signaling activities of the NLRP3 and ASC-containing inflammasome," Journal of Immunology, vol. 182, no. 10, pp. 6460-6469, 2009.

[94] S. B. Willingham, I. C. Allen, D. T. Bergstralh et al., "NLRP3 (NALP3, cryopyrin) facilitates in vivo caspase-1 activation, necrosis, and HMGB1 release via inflammasome-dependent and -independent pathways," Journal of Immunology, vol. 183, no. 3, pp. 2008-2015, 2009.

[95] A. A. Abdul-Sater, N. Said-Sadier, E. V. Padilla, and D. M. Ojcius, "Chlamydial infection of monocytes stimulates IL-1 $\beta$ secretion through activation of the NLRP3 inflammasome," Microbes and Infection, vol. 12, no. 8-9, pp. 652-661, 2010.

[96] X. He, S. Mekasha, N. Mavrogiorgos, K. A. Fitzgerald, E. Lien, and R. R. Ingalls, "Inflammation and fibrosis during Chlamydia pneumoniae infection is regulated by IL-1 and the NLRP3/ASC inflammasome," Journal of Immunology, vol. 184, no. 10, pp. 5743-5754, 2010. 
[97] I. E. Brodsky, N. W. Palm, S. Sadanand et al., "A Yersinia effector protein promotes virulence by preventing inflammasome recognition of the type III secretion system," Cell Host and Microbe, vol. 7, no. 5, pp. 376-387, 2010.

\section{This article should be cited as follows:}

Manoranjan Sahoo, Ivonne Ceballos-Olvera, Laura del Barrio, and Fabio Re, "Role of the Inflammasome, IL-1 $\beta$, and IL-18 in Bacterial Infections," TheScientificWorldJOURNAL, vol. 11, pp. 2037-2050, 2011. 


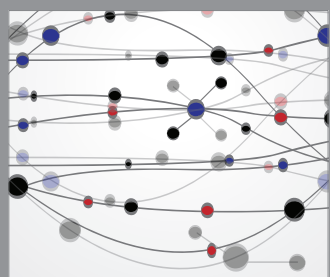

The Scientific World Journal
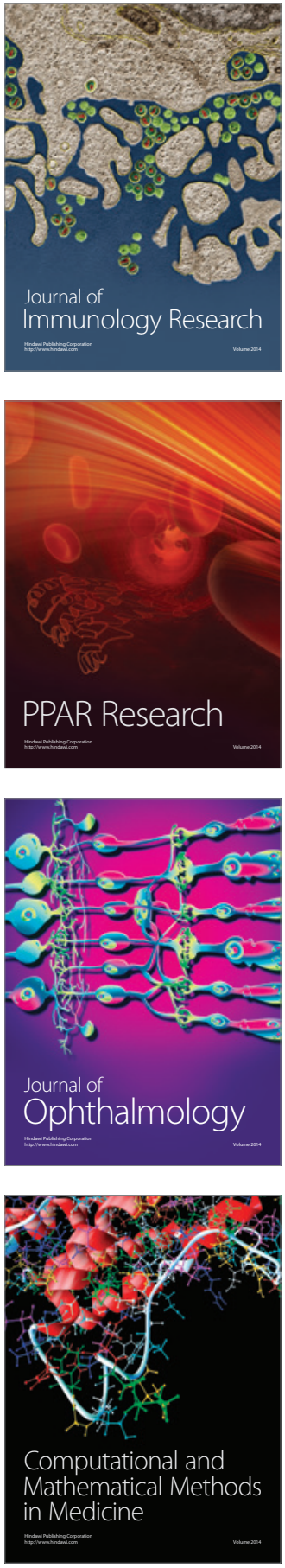

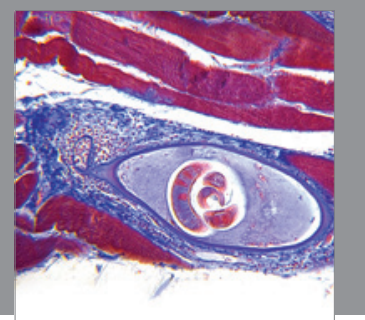

Gastroenterology

Research and Practice
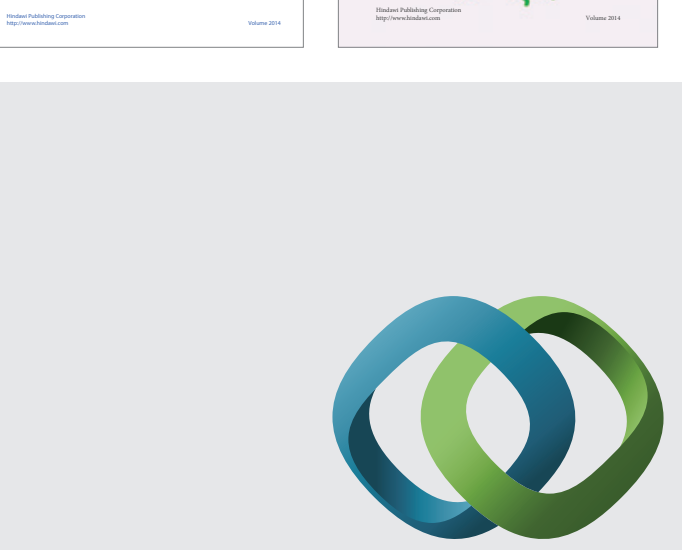

\section{Hindawi}

Submit your manuscripts at

http://www.hindawi.com
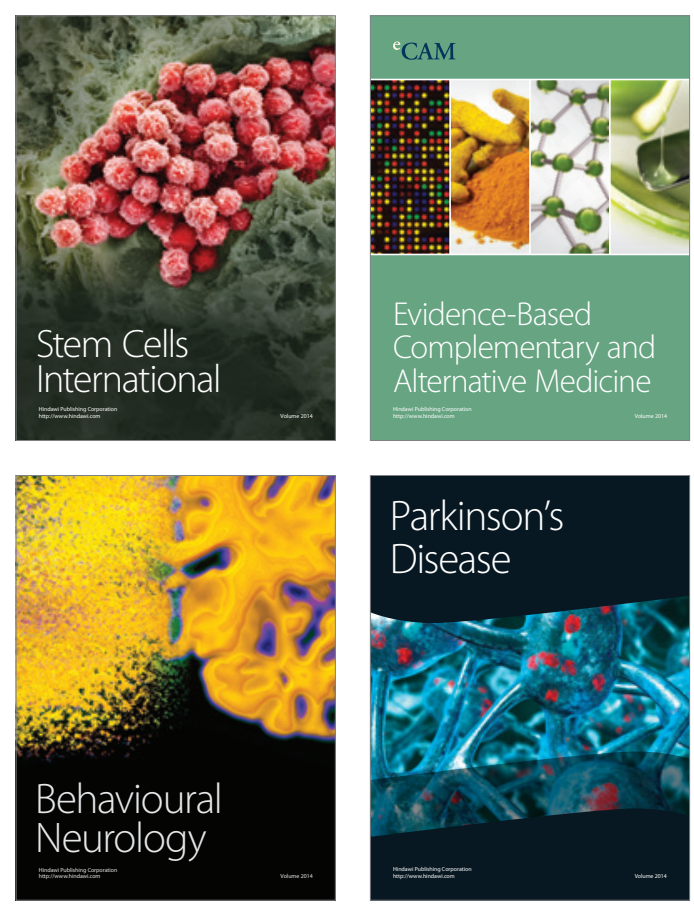

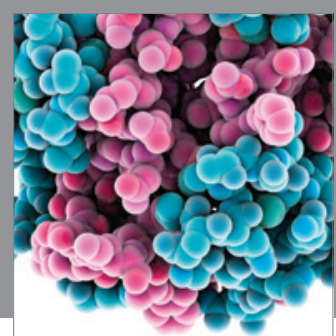

Journal of
Diabetes Research

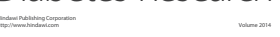

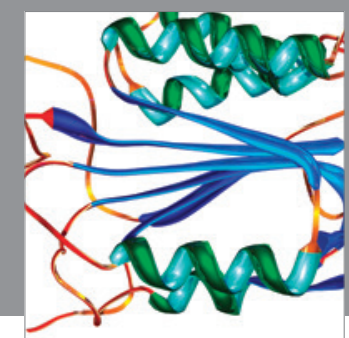

Disease Markers
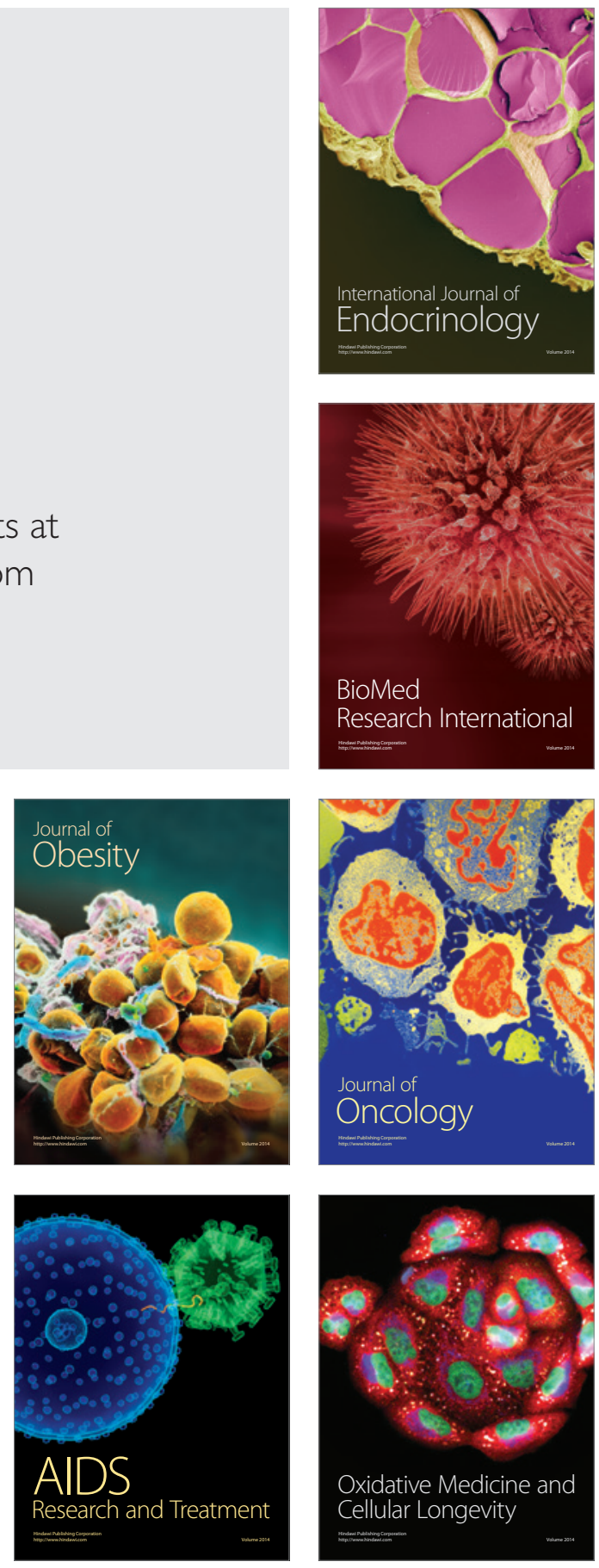